\title{
ALIMENTACIÓN Y NUTRICIÓN EN CÁNCER HEMATOLÓGICO: DISEÑO Y VALIDACIÓN DE UNA INFOGRAFÍA PSICOEDUCATIVA
}

\section{Feeding and nutrition in hematological cancer: design and validation of a psychoeducational infographic}

\author{
Lindsay Carrillo Valdez ${ }^{\mathrm{a}}$ y María Luisa Escamilla Gutiérrez
}

Recibido: 3 de enero, $2020 \bullet$ Aprobado: 22 de abril, 2020

Cómo citar: Carrillo Valdez L, Escamilla Gutiérrez ML. Alimentación y nutrición en cáncer hematológico: diseño y validación de una infografía psicoeducativa. cysa [Internet]. 18 de septiembre de 2020 [citado 18 de septiembre de 2020];4(3):13-21. Disponible en: https://revistas.intec.edu.do/index.php/cisa/article/view/1923

\section{Resumen}

El cáncer y su tratamiento implican cambios en la dieta y metabolismo que aumentan el riesgo de desarrollar desnutrición, especialmente cuando los pacientes desconocen cómo alimentarse. Aunque se han desarrollado materiales psicoeducativos para favorecer conductas alimentarias saludables, la mayoría son extensos, están dirigidos a pacientes diagnosticados con tumores sólidos o no señalan datos de validación. Con el objetivo de construir y validar un material psicoeducativo breve, orientado a la promoción de conductas alimentarias y nutricionales saludables del paciente oncohematológico adulto, se diseñó y validó la infografía Recomendaciones para la alimentación del paciente oncohematológico. Se empleó la metodología propuesta por la Organización Panamericana de la Salud y por Ziemendorff y Krause en un grupo de jueces expertos $(\mathrm{n}=18)$ y otro de pacientes oncohematológicos $(\mathrm{n}=20)$. Se obtuvieron acuerdos porcentuales superiores a $80 \%$ y $x>.68$ en ambos grupos de validación, por lo tanto, la infografía diseñada es un instrumento conciso, válido y específico que puede ser distribuida entre la población diana.

Palabras clave: cáncer; hematología; conducta alimentaria; calidad de vida; educación en salud.

\footnotetext{
a Maestría en Psicología, Universidad Nacional Autónoma de México, México. Programa de Maestría y Doctorado. Facultad de Estudios Superiores Iztacala. Correo-e: psiclcarrillovz@gmail.com
}

\begin{abstract}
Cancer and its treatment involve changes in diet and metabolism that increase the risk of developing malnutrition, especially when patients do not know how to feed. Although psychoeducational materials have been developed to favor healthy eating behaviors, the majority are extensive, they are aimed at patients diagnosed with solid tumors or do not indicate validation data. In order to create and validate a brief psychoeducational material aimed at promoting healthy eating and nutritional behaviors of the adult oncohematological patient, the infographic Recommendations for feeding the oncohematological patient was designed and validated. The methodology proposed by the Pan American Health Organization and by Ziemendorff and Krause was used in a group of expert judges ( $\mathrm{n}=$ 18) and another group of oncohematological patients ( $\mathrm{n}$ $=20$ ). Percent agreements greater than $80 \%$ and $x>.68$ were obtained in both validation groups, therefore, the designed infographic is a concise, valid and specific instrument that can share out among the target population.
\end{abstract}

Keywords: cancer; hematology; feeding behavior; quality of life; health education.

\footnotetext{
b Maestría en Psicología, Universidad Nacional Autónoma de México, Programa de Maestría y Doctorado. Facultad de Estudios Superiores Iztacala. Correo-e: marilu.escamilla16@gmail.com
} 


\section{Introducción}

El cáncer es una de las principales causas de morbilidad y mortalidad en el mundo. ${ }^{1}$ Tan solo en 2018 se reportaron 18078957 casos nuevos y cobró la vida de 9555027 pacientes. En lo que respecta a las neoplasias hematológicas malignas, se presentaron 1186598 incidencias y 690002 fallecimientos. ${ }^{2}$ La atención integral de los diferentes tipos de cáncer en la sangre es un reto importante por su impacto epidemiológico en poblaciones cada vez más jóvenes, ${ }^{3}$ así como las consecuencias biopsicosociales que experimentan pacientes, cuidadores $\mathrm{y}$ sistemas sanitarios. ${ }^{4-6}$

Tras un diagnóstico oncohematológico, actividades cotidianas como comer, se convierten en un desafío, especialmente cuando los pacientes no cuentan con conocimientos en alimentación y nutrición que favorezcan conductas saludables. ${ }^{7}$ De ahí que uno de los principales motivos de preocupación de pacientes y cuidadores esté relacionado con los cambios y dificultades en la dieta, derivados de la enfermedad, su localización y secuelas en el organismo, además de las interacciones y efectos secundarios del tratamiento, que aumentan el riesgo de desarrollar síndromes paraneoplásicos como anorexia, desnutrición, caquexia u obesidad con sarcopenia, los cuales se presentan al menos en el $50 \%$ de los pacientes oncohematológicos. ${ }^{8-18}$

Pese a que la asesoría dietética ha demostrado efectos positivos en el mantenimiento del peso corporal, masa muscular, tejido adiposo, ${ }^{15,19-22}$ calidad de vida ${ }^{23}$ y funcionamiento emocional de los pacientes oncológicos, ${ }^{22}$ es poco frecuente su detección, evaluación y canalización tempranas. ${ }^{17,19,21,22}$ Además, el advenimiento de dietas alternativas (por ejemplo, la dieta cetogénica), ${ }^{24}$ que los pacientes adoptan, por mitos y creencias, ${ }^{25,}{ }^{26}$ sin ningún tipo de notificación, vigilancia o evidencia científica de sus beneficios, ${ }^{8,21,27}$ ha contribuido a que los problemas de alimentación-nutrición sean más frecuentes, empero pasen desapercibidos por el equipo sanitario.
En consecuencia, la mayoría de las intervenciones nutricionales se enfocan en los niveles secundario y terciario de prevención, cuando el riesgo o estado de desnutrición son evidentes. ${ }^{8-11,15,18-23}$ Por lo tanto, resulta importante el desarrollo de intervenciones que favorezcan el acceso temprano al asesoramiento nutricional individualizado que, mediante estrategias y materiales diversos, coadyuven a la resolución del problema de información insuficiente y dudas que reportan pacientes y cuidadores, faciliten la sensación de control y promuevan el acercamiento a especialistas en dieta, nutrición y cáncer desde el diagnóstico y a lo largo del tratamiento médico. ${ }^{8-12,14,15,19-23}$

Actualmente, existen libros, guías y manuales, que incluyen pautas para la alimentación-nutrición de los pacientes oncológicos ${ }^{12,19,21-22,28,29}$ y oncohematológicos, ${ }^{30}$ empero se trata de materiales muy extensos $^{31}$ en los que, además, no se identifican datos de validación y/o adaptación cultural en el público meta. En cuanto a los materiales breves, como infografías, la mayoría se encuentran en sitios de internet poco confiables, mientras que aquellos avalados por autoridades e instituciones de salud solo están disponibles para cánceres sólidos. ${ }^{32}$ Por tanto, el objetivo de la presente investigación fue construir y validar un material psicoeducativo breve orientado a la promoción de conductas alimentarias-nutricionales saludables en el paciente oncohematológico adulto.

\section{Materiales y métodos}

Se aplicó la metodología para validación de herramientas de comunicación y materiales educativos propuesta por la Organización Panamericana de la Salud (OPS) ${ }^{33}$ y por Ziemendorff y Krause. ${ }^{34}$

La fase preliminar consistió en la elección del mensaje a partir de la revisión de literatura especializada. Posteriormente, se construyó una infografía en el sitio web de diseńo gráfico y acceso libre 
Canva, fue descargada en formato PDF e impresa a color en multifuncional HP Deskjet $2510^{\circ}$, en hoja de papel bond blanco (93\%), tamaño carta $(21.59 \times 27.94 \mathrm{~cm})$.

El material, titulado Recomendaciones para la alimentación del paciente oncohematológico, señala los macro y micronutrientes y sus funciones, así como los grupos de alimentos que integran una dieta adecuada. También, se enlistan conductas alimentarias que coadyuvan al manejo de síntomas derivados del cáncer hematológico y su tratamiento. Asimismo, se proporciona la dirección electrónica de la American Cancer Society (en español) para acceder a información complementaria. Finalmente, se destaca la importancia de consultar al experto en Nutrición.

El equipo de investigación evaluó el material mediante los Criterios generales para todo tipo de material de la OPS. ${ }^{33}$ Seguidamente, para la fase de validación por jueces, se conformaron dos grupos a partir de un muestreo intencional no probabilístico. Los jueces expertos (médicos y nutriólogos) fueron ubicados en sus espacios de trabajo, mientras que los jueces no expertos (pacientes oncohematológicos) pertenecían al servicio de Hematología de un hospital de la Ciudad de México. Sus principales características sociodemográficas se resumen en la tabla 1.

Tabla 1. Características sociodemográficas de los grupos de jueces expertos y no expertos

\begin{tabular}{|c|c|c|c|c|c|c|c|c|c|c|c|c|c|c|c|c|c|c|c|}
\hline \multirow{5}{*}{$\begin{array}{c}\text { Grupo } \\
\\
\text { Jueces } \\
\text { expertos } \\
(\mathrm{n}=18)\end{array}$} & \multicolumn{19}{|c|}{ Variable } \\
\hline & \multicolumn{4}{|c|}{ Sexo } & \multicolumn{3}{|c|}{ Edad } & \multicolumn{8}{|c|}{ Especialidad } & \multirow{2}{*}{\multicolumn{4}{|c|}{$\begin{array}{l}\text { Atención de } \\
\text { pacientes } \\
\text { oncológicos }\end{array}$}} \\
\hline & \multicolumn{2}{|c|}{ M } & \multicolumn{2}{|c|}{$\mathrm{H}$} & \multirow{2}{*}{ Rango } & \multirow{2}{*}{$\mathrm{ME}$} & \multirow{2}{*}{$\mathrm{DE}$} & \multicolumn{2}{|c|}{ HM } & \multicolumn{2}{|c|}{ MF } & \multicolumn{2}{|c|}{ MP } & \multicolumn{2}{|c|}{ NT } & & & & \\
\hline & $\mathrm{f}$ & $\%$ & $\mathrm{f}$ & $\%$ & & & & $\mathrm{~F}$ & $\%$ & $\mathrm{f}$ & $\%$ & F & $\%$ & $\mathrm{f}$ & $\%$ & \multirow{2}{*}{\multicolumn{4}{|c|}{ Sí }} \\
\hline & 16 & 89 & 2 & 11 & $26-56$ & 35.6 & 8.9 & 5 & 28 & 8 & 44 & 1 & 5.6 & 4 & 22 & & & & \\
\hline \multirow{4}{*}{$\begin{array}{l}\text { Jueces no } \\
\text { expertos } \\
(\mathrm{n}=20)\end{array}$} & \multicolumn{4}{|c|}{ Sexo } & \multicolumn{3}{|c|}{ Edad } & \multicolumn{12}{|c|}{ Enfermedad } \\
\hline & \multicolumn{2}{|c|}{ M } & \multicolumn{2}{|c|}{$\mathrm{H}$} & \multirow{2}{*}{ Rango } & \multirow{2}{*}{$\mathrm{ME}$} & \multirow{2}{*}{$\mathrm{DE}$} & \multicolumn{2}{|c|}{ LNH } & \multicolumn{2}{|c|}{ LH } & \multicolumn{2}{|c|}{ LMA } & \multicolumn{2}{|c|}{ LLA } & \multicolumn{2}{|c|}{ MM } & \multicolumn{2}{|c|}{ LLC } \\
\hline & $\mathrm{f}$ & $\%$ & $\mathrm{f}$ & $\%$ & & & & $\mathrm{~F}$ & $\%$ & $\mathrm{f}$ & $\%$ & $\mathrm{~F}$ & $\%$ & $\mathrm{f}$ & $\%$ & $\mathrm{f}$ & $\%$ & $\mathrm{f}$ & $\%$ \\
\hline & 11 & 55 & 9 & 45 & $19-72$ & 42.9 & 16.5 & 8 & 40 & 3 & 15 & 3 & 15 & 3 & 15 & 2 & 10 & 1 & 5 \\
\hline
\end{tabular}

Fuente: elaboración propia.

Nota: $\mathrm{HM}=$ Hematología; $\mathrm{MF}=$ Medicina Familiar; $\mathrm{MP}=$ Medicina Preventiva; NT=Nutrición. LNH=Linfoma no Hodgkin; LH=Linfoma de Hodgkin; LMA=Leucemia Mieloide Aguda; LLA=Leucemia Linfoide Aguda; MM=Mieloma Múltiple; LLC=Leucemia Linfocítica Crónica. 
Los especialistas recibieron de manera personal la infografía impresa y la rúbrica Criterios especificos para material escrito de la OPS. ${ }^{33}$ Se obtuvieron respuestas y recomendaciones escritas. Por otro lado, para el grupo de pacientes, tras la entrega del material impreso, se realizaron entrevistas personales, ${ }^{33,34}$ basadas en la misma rúbrica empleada para jueces expertos, y se aplicó el Cuestionario final sobre formato, preferencia y proyección de la OPS. ${ }^{33}$

El análisis de datos consistió en la obtención del porcentaje de acuerdo entre los evaluadores, para cada uno de los criterios de la rúbrica y cuestionario aplicados, así como el cálculo del índice
Kappa de Fleiss con el IBM-SPSS, versión 26 para ambos grupos.

\section{Resultados}

En la fase preliminar se cumplieron los criterios generales para el diseño y la utilización de materiales con fines educativos definidos por la OPS. Asimismo, en la evaluación inicial, se obtuvieron 11 de 12 Criterios generales para todo tipo de material $^{3}$ (véase tabla 2), que permitió continuar con el proceso de validación de la infografía psicoeducativa.

Tabla 2. Criterios generales para toda clase de material cumplidos por el material psicoeducativo

\begin{tabular}{|l|c|c|}
\hline \multicolumn{1}{|c|}{ Criterio } & Cumple & $\begin{array}{c}\text { No } \\
\text { cumple }\end{array}$ \\
\hline 1. ¿Participó el público objetivo en la selección, elaboración y evaluación del material? & $\mathrm{X}$ & \\
\hline 2. ¿Representa situaciones de la vida diaria? & $\mathrm{X}$ & \\
\hline 3. ¿Forman parte de un programa de comunicación o educación? & & $\mathrm{X}$ \\
\hline 4. ¿Están disponibles los servicios o recursos que promueve? & $\mathrm{X}$ & \\
\hline 5. ¿Se dispone de otros materiales y técnicas que refuercen los mensajes? & $\mathrm{X}$ & \\
\hline 6. ¿Han sido sometidos a prueba antes de su elaboración final? & $\mathrm{X}$ & \\
\hline 7. ¿Hay instructivos para su utilización? & $\mathrm{X}$ & \\
\hline $\begin{array}{l}\text { 8. ¿Se ha definido el público al que va dirigido en términos de nivel educativo, } \\
\text { características culturales, geográficas y socioeconómicas? }\end{array}$ & $\mathrm{X}$ & \\
\hline $\begin{array}{l}\text { 9. ¿Se ha cuidado que el material no contenga elementos ofensivos para las tradiciones } \\
\text { de la comunidad? }\end{array}$ & $\mathrm{X}$ & \\
\hline $\begin{array}{l}\text { 10. ¿Es evidente que el personal o técnicos de salud tuvieron participación en su } \\
\text { diseño? }\end{array}$ & $\mathrm{X}$ & \\
\hline 11. ¿Respeta la libertad de decisión del público sin manipularlo? & $\mathrm{X}$ & $\mathrm{X}$ \\
\hline 12. ¿Responde a un objetivo específico? & \\
\hline
\end{tabular}

Fuente: elaboración propia.

16 | Ciencia y Salud 2020; 4(3, septiembre-diciembre): Artículo original • 13-21 
Todos los jueces expertos proporcionaron calificaciones totales entre 41 y 45 puntos en los Criterios especificos para material escrito de la OPS, ${ }^{33}$ por lo que el mensaje de la infografía no tuvo que ser modificado, ni sometido a otra fase de validación. Ahora bien, el porcentaje de acuerdo para este grupo fue superior al $80 \%$ en todos los ítems (véase tabla 3). Adicionalmente, se obtuvo el coeficiente Kappa de Fleiss con el fin de excluir concordancias debidas al azar. La fuerza de concordancia resultó considerable ${ }^{34-37}(x=.682)$ y el material se aceptó como válido.

Tabla 3. Porcentaje de acuerdo entre jueces expertos por criterio evaluado

\begin{tabular}{|c|c|}
\hline Criterio & $\%$ \\
\hline 1. Presenta un tema específico en forma completa. & 83.3 \\
\hline $\begin{array}{l}\text { 2. El contenido del mensaje es fácilmente } \\
\text { comprensible. }\end{array}$ & 82.2 \\
\hline $\begin{array}{l}\text { 3. Las ilustraciones aclaran o complementan lo } \\
\text { escrito. }\end{array}$ & 100 \\
\hline 4. El tamaño de la letra facilita la lectura. & 100 \\
\hline $\begin{array}{l}\text { 5. Hay elementos de síntesis del mensaje o } \\
\text { contenido. }\end{array}$ & 84.4 \\
\hline $\begin{array}{l}\text { 6. Existen elementos para resaltar ideas } \\
\text { importantes, tales como subrayado, marcación de } \\
\text { letras con otros colores, etcétera. }\end{array}$ & 100 \\
\hline $\begin{array}{l}\text { 7. La ortografía, gramática, puntuación y redacción } \\
\text { son adecuadas. }\end{array}$ & 100 \\
\hline 8. No está recargado de información escrita. & 100 \\
\hline 9. Usa lenguaje comprensible para el público. & 83.3 \\
\hline
\end{tabular}

Fuente: elaboración propia.
De forma similar, los jueces no expertos otorgaron entre 40 y 43 puntos totales al material, por lo que permaneció sin cambios. En cuanto al porcentaje de acuerdo obtenido para este grupo fue igual o mayor al $80 \%$ (véase tabla 4) y $x=.742$ (considerable). ${ }^{34-37}$ por lo que el diseńo y mensaje se aceptaron como válidos.

Tabla 4. Porcentaje de acuerdo entre jueces no expertos por criterio evaluado

\begin{tabular}{|l|c|}
\hline \multicolumn{1}{|c|}{ Criterio } & $\%$ \\
\hline 1. Presenta un tema específico en forma completa. & 98 \\
\hline $\begin{array}{l}\text { 2. El contenido del mensaje es fácilmente } \\
\text { comprensible. }\end{array}$ & 81 \\
\hline $\begin{array}{l}\text { 3. Las ilustraciones aclaran o complementan lo } \\
\text { escrito. }\end{array}$ & 98 \\
\hline \begin{tabular}{l} 
4. El tamaño de la letra facilita la lectura. \\
\hline $\begin{array}{l}\text { 5. Hay elementos de síntesis del mensaje o } \\
\text { contenido. }\end{array}$
\end{tabular} & 81 \\
\hline $\begin{array}{l}\text { 6. Existen elementos para resaltar ideas } \\
\text { importantes, tales como subrayado, marcación de } \\
\text { letras con otros colores, etcétera. }\end{array}$ & 100 \\
\hline $\begin{array}{l}\text { 7. La ortografía, gramática, puntuación y } \\
\text { redacción son adecuadas. }\end{array}$ & 100 \\
\hline 8. No está recargado de información escrita. & 87 \\
\hline 9. Usa lenguaje comprensible para el público. & 81 \\
\hline
\end{tabular}

Fuente: elaboración propia.

De acuerdo con los datos obtenidos en el Cuestionario final sobre formato, preferencia y proyección de la OPS, ${ }^{33}$ la infografía resultó agradable, novedosa, importante y útil para todos los jueces no expertos. Consideraron que el material puede ser distribuido entre pacientes, familiares, amigos, enfermeras y médicos tratantes, ya que resuelve dudas, brinda 
sugerencias prácticas sobre la dieta y el manejo de efectos secundarios del tratamiento, que hasta el momento habían realizado sin orientación formal, e incita a acudir al nutriólogo como parte del tratamiento integral de su enfermedad. Finalmente, se midieron, a manera de proyección, las variables trascendencia, probabilidad de éxito y vinculación al objetivo principal ${ }^{4}$ y se calculó la eficacia esperada para el material psicoeducativo. Se obtuvieron 80 puntos $(64 \%)$ de 125 posibles, un resultado aceptable.

\section{Discusión}

El objetivo del presente trabajo fue construir y validar un material psicoeducativo breve, orientado a la promoción de conductas alimentarias-nutricionales saludables en el paciente oncohematológico adulto. Se eligió una infografía debido a que se trata de un instrumento conciso, económico y didáctico, ampliamente utilizado en las Ciencias de la Salud por la preferencia del público, su contribución a la comunicación entre especialistas, pacientes y cuidadores y su capacidad de promoción de cambios comportamentales, ${ }^{38}$ tal como se observó en este estudio.

El proceso de validación de la infografía psicoeducativa permitió constatar lo señalado por la literatura respecto de la preocupación por los cambios en la dieta tras un diagnóstico oncológico; $;^{8-18}$ la realización de conductas alimentarias de riesgo, 8,21,25-27 como "no comer o comer menos" (sic. pac.), "evitar la carne y el azúcar" (sic. pac.), "comer solo frutas y verduras" (sic. pac.), entre otras derivadas de la falta de información y acceso a servicios nutricionales $^{8-12,14,15,17,19-23}$ que coloca a los pacientes en riesgo de desarrollar comorbilidades que afectarían su calidad de vida, tratamiento médico y pronóstico. $^{8-17,19-23}$

Así, por su especificidad, capacidad de atracción, comprensión, aceptación, utilidad, importancia y validez ${ }^{33,34}$ es posible proceder a la distribución masiva del material Recomendaciones para la alimentación del paciente oncohematológico que permitirá responder a las necesidades educativas alimentario-nutricionales de los pacientes oncohematológicos y prevenir, en un nivel primario, las complicaciones asociadas a lo largo del curso de la enfermedad.

No obstante, se debe considerar la interacción del factor novedad en la obtención de los resultados aquí presentados en atención a que: 1) no existen muchos materiales similares; 2) en la muestra contemplada, ningún juez experto había participado en el diseńo y evaluación de instrumentos psicoeducativos para la salud; y 3) ningún paciente había recibido indicaciones dietéticas personalizadas y formales más allá de la indicación médica "comer de todo" (sic. pac.).

Por último, es importante señalar que la trascendencia potencial del material fue medida con los datos de incidencia y prevalencia de cánceres hematológicos en México para $2018^{2}$ como línea base; sin embargo, la muestra de pacientes de esta investigación $(\mathrm{n}=20)$ es muy pequeña comparada con la población diana (15 610 casos nuevos y 45063 casos prevalentes). ${ }^{2}$ Por tanto, es necesario someter la infografía a más estudios de validación y eficacia en muestras más grandes.

\section{Conclusiones}

El diseńo de materiales psicoeducativos es imprescindible para la asimilación de información en salud, su comprensión y práctica. La infografía presentada en este estudio es un instrumento conciso, válido, específico y eficaz en la promoción de conductas alimentarias-nutricionales saludables entre los pacientes oncohematológicos adultos. Sin embargo, no es la solución a las problemáticas complejas de alimentación y nutrición en cáncer. Por consiguiente, es indispensable que se le considere dentro de un programa educa- 
tivo oportuno, inclusivo e interdisciplinario que garantice: a) el derecho al acceso a la educación en salud; b) la difusión masiva a través de todos los medios disponibles, incluidas las nuevas Tecnologías de la Información y Comunicación; ${ }^{39}$ y c) asegure la adherencia terapéutica y mantenimiento de las conductas saludables a lo largo de las etapas de diagnóstico, tratamiento, vigilancia, recidivas e incluso en fase terminal. ${ }^{8,14}$

\section{Referencias}

1. World Health Organization. WHO: Cancer [online]. Available from: https://www.who. int/es/news-room/fact-sheets/detail/cancer [Accessed 2nd December 2019]

2. International Agency for Research on Cancer, World Health Organization. IARC and $\mathrm{WHO}$. The global cancer observatory [online]. Available from: http://gco.iarc.fr/ today/home [Accessed 2nd December 2019]

3. Tirado-Gómez LL, Mohar A. Epidemiología de las neoplasias hemato-oncológicas. Cancerología. 2007;2:109-20.

4. Reynoso-Noverón N, Torres-Domínguez JA. Epidemiología del cáncer en México. Carga global y proyecciones 2000-2020. Rev Latinoam Med Conducta. 2017;8(1):9-15.

5. Ríos-Quezada MJ, Cruzat-Mandich, C. Percepción y significados asociados a la calidad de vida en pacientes hemato-oncológicos. Rev Chil Neuro-Psiquiat. 2015;53(4):261-8.

6. Carbajal C. El impacto del diagnóstico médico como experiencia traumática. Algunas reflexiones. Rev Med Clin Condes. 2017;28(6):841-8. Doi: 10.1016/j. rmclc.2017.10.010
7. Hawrysz I, Krusińska B, Słowińska MA, Wądołowska L, Czerwińska A, Biernacki M. Nutritional knowledge, diet quality and breast or lung cancer risk: a case-control study of adults from Warmia and Mazury region in Poland. Rocz Panstw Zakl Hig. 2016;67(1):9-15.

8. Carvalho G, Camilo ME, Ravasco P. Qual a relevância da nutriçãa em oncología. Acta Med Port. 2011;24(S4):1041-50.

9. Coa KI, Epstein JB, Ettinger D, et al. The impact of cancer treatment on the diets and food preferences of patients receiving outpatient treatment. Nutr Cancer. 2015;67(2):339-53. Doi:10.1080/0163558 1.2015 .990577

10. Demoor-Goldschmidt C, Raynard B. Comment intégrer la prise en charge nutritionnelle au projet thérapeutique en cancérologie? Bull Cancer. 2009;96(6):665-75. Doi:10.1684/bdc.2009.0887

11. Gangadharan A, Choi SE, Hassan A, et al. Protein calorie malnutrition, nutritional intervention and personalized cancer care. Oncotarget. 2017;8(14):24009-30. Doi: 10. 18632/oncotarget. 15103

12. Peña MG. Dieta y cáncer. En: Goméz Candela C, Sastre Gallego A, editores. Soporte nutricional en el paciente oncológico. Madrid: You\&Us; 2004. p. 115-131.

13. Hopkinson J, Wright D, Corner J. Exploring the experience of weight loss in people with advanced cancer. Available from: https:// www.ncbi.nlm.nih.gov/pubmed/16629915" lo "Journal of advanced nursing. 2006;54(3): 304-12 
14. Hopkinson J. Psychosocial support in cancer cachexiasyndrome: The evidenceforsupported self-management of eating problems during radiotherapy or chemotherapy treatment. Asia Pac J Oncol Nurs. 2018;5(4):358-68. Doi:10.4103/apjon.apjon_12_18

15. Ravasco P, Monteiro-Grillo I, Marques P, Camilo, M. Dietary counseling improves patient outcomes: a prospective, randomized, controlled trial in colorectal cancer patients undergoing radiotherapy. J Clin Oncol. 2005;23(7):1431-8.

16. Sendrós MJ. Nutr Hosp. Aspectos dietoterapéuticos en situaciones especiales del paciente oncológico. Prebióticos y probióticos, ¿̇tienen cabida en la terapia nutricional del paciente oncológico? 2016;33(Supl. 1):17-30.

17. Spiro A, Baldwin C, Patterson A, Thomas J, Andreyev HJ. The views and practice of oncologists towards nutritional support in patients receiving chemotherapy. $\mathrm{Br} \mathrm{J}$ Cancer. 2006;95(4):431-4. Doi:10.1038/ sj.bjc. 6603280

18. Baltazar-Luna E, Bravo-Álvarez G, Sámano R, Chico-Barba G. Utilidad del CONUT frente al NRS-2002 en la valoración de riesgo nutricional en pacientes hemato-oncológicos. Nutr Clín Diet Hosp. 2017;37(1):1723. Doi: $10.12873 / 371$ baltazarluna

19. Cotogni P, Pedrazzoli P, De Waele E, et al. Nutritional therapy in cancer patients receiving chemoradiotherapy: Should we need stronger recommendations to act for improvingOutcomes?JCancer.2019;10(18):431825. Doi: $10.7150 /$ jca. 31611

20. Aoyama T, Yoshitsugu K, Fukaya M, el al. Benefit of reducing body weight loss with a nutritional support pathway in patients undergoing allogeneic hematopoietic stem cell transplantation. Med Sci Monit Basic Res. 2019;25:187-98. Doi: 10.12659/ MSMBR.917329

21. Caccialanza R, Pedrazzoli P, Cereda E, et al. Nutritional support in cancer patients: A position paper from the Italian Society of Medical Oncology (AIOM) and the Italian Society of Artificial Nutrition and Metabolism (SINPE). J Cancer. 2016;7(2):131-5. Doi: $10.7150 /$ jca. 13818 .

22. Hopkinson JB. The nursing contribution to nutritional care in cancer cachexia. Proc Nutr Soc. 2015;74(4):413-8. Doi: 10.1017/ S0029665115002384

23. Baldwin C, Spiro A, Ahern R, Emery PW. Oral nutritional interventions in malnourished patients with cancer: a systematic review and meta-analysis. J Natl Cancer Inst. 2012;104(5):371-85. Doi: 10.1093/jnci/ djr556

24. O'Flanagan $\mathrm{CH}$, Smith LA, McDonell SB, Hursting SD. When less may be more: calorie restriction and response to cancer therapy. BMC Med. 2017;15(1):106. Doi: 10.1186/s12916-017-0873-x.

25. Murillo-Godínez G, Pérez-Escamilla LM. Los mitos alimentarios y su efecto en la salud humana. Med Int Méx. 2017;33(3): 392-402.

26. Fundació Alícia. Institut Català d'Oncologia. Mitos y creencias sobre la alimentación durante el tratamiento del cáncer. Comer durante el tratamiento del cáncer [online]. Catalunya: Fundación Catalunya-La Pedrera; 2016 [Accessed 2nd December 2019]. Available from: http://ico.gencat.cat/web/.content/ minisite/ico/ciutadans/documents/arxius/ Mitos-y-creencias-sobre-la-alimentacion-durante-el-tratamiento-del-cancer.pdf 
27. Caccialanza R, Aprile G, Cereda E. et al. Fasting in oncology: a word of caution. Nat Rev Cancer. 2019;19(177). Doi:10.1038/ s41568-018-0098-0

28. Martínez JR, Villarino A. Recomendaciones de alimentación para pacientes oncológicos. Avances en alimentación, nutrición y dietética [online]. Madrid: Fundación Alimentación Saludable; 2015 [Accessed 1st March 2019]. Available from: https://www.aecc.es/ sites/default/files/content-file/Recomendaciones\%20_Alimentacion.pdf

29. Salces M, Paz R., Hernández-Navarro F. Recomendaciones nutricionales en el paciente oncohematológico. Nutr Hosp. 2006;21(3):379-85.

30. Martínez JR, Villarino A. Recomendaciones de alimentación para pacientes oncológicos. Avances en alimentación, nutrición y dietética [online]. Madrid: Fundación Alimentación Saludable; 2015 [Accessed 1st March 2019]. Available from: https://www.aecc.es/ sites/default/files/content-file/Recomendaciones\%20_Alimentacion.pdf

31. Gómez C, Barragán B, Díaz J, et al. AEAL Explica: Recomendaciones en alimentación y nutrición para pacientes oncohematológicos [online]. Madrid: AEAL. Asociación Española de Afectados por Linfoma, Mieloma y Leucemia; 2011 [Accessed 9th January 2019]. Available from: http://www.aeal.es/ nueva_web/wp-content/uploads/2015/07/ aeal_explica_alimentacion_nutricion.pdf

32. Infocancer. Nacional de Cancerología. INCan: Nutrición para pacientes con cáncer de mama [online]. Available from: https://www.infocancer.org.mx/?i=infografia\&a=nutricion-pacientes-cancer [Accessed 1st December 2019].
33. Organización Panamericana de la Salud. Organización Mundial de la Salud. Herramientas de comunicación para el desarrollo de entornos saludables. 2006. Washington, DC: Organización Panamericana de la Salud.

34. Ziemendorff S., Krause L. Guía de validación de materiales educativos (con enfoque en materiales de Educación Sanitaria). 2005. Chiclayo: Programa de Agua y Saneamiento PROAGUA/GTZ.

35. Cerda J, Villarroel L. Evaluación de la concordancia inter-observador en investigación pediátrica: Coeficiente de Kappa. Rev Chil Pediatr. 2008;79(1),54-8.

36. Torres JJ, Perera VH. Cálculo de la fiabilidad y concordancia entre codificadores de un sistema de categorías para el estudio del foro online en e-learning. Revista de Investigación Educativa. 2009;27(1),89-103.

37. Vio F, Lera L, Fuentes-García A, Salinas J. Método Delphi para identificar materiales educativos sobre alimentación saludable para educadores, escolares y sus padres. Archivos latinoamericanos de nutrición. 2012;62(3):275-82.

38. Sanz-Lorente M, Castejón-Bolea R. Infografías en las ciencias de la salud: aplicación al cuidado domiciliario. Hosp Domic. 2018;2(2):67-78.

39. Coughlin SS, Whitehead M, Sheats JQ, et al. Smartphone applications for promoting healthy diet and nutrition: a literature review. Jacobs J Food Nutr. 2015;2(3):21-35. 